\title{
Multi-centre evaluation of real-time multiplex PCR for detection of carbapenemase genes OXA-48, VIM, IMP, NDM and KPC
}

\author{
Anneke van der Zee ${ }^{1 *}$, Lieuwe Roorda', Gerda Bosman ${ }^{1}$, Ad C Fluit ${ }^{2}$, Mirjam Hermans ${ }^{3}$, Paul HM Smits ${ }^{4}$, \\ Adri GM van der Zanden ${ }^{5}$, René te Witt ${ }^{6}$, Lesla ES Bruijnesteijn van Coppenraet ${ }^{7}$, James Cohen Stuart ${ }^{8}$ \\ and Jacobus M Ossewaarde ${ }^{1,6}$
}

\begin{abstract}
Background: Resistance to carbapenem antibiotics is emerging worldwide among Enterobacteriaceae. To prevent hospital transmission due to unnoticed carriage of carbapenemase producing micro-organisms in newly admitted patients, or follow-up of patients in an outbreak setting, a molecular screening method was developed for

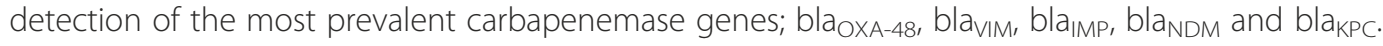

Methods: A real-time multiplex PCR assay was evaluated using a collection of $86 \mathrm{Gram}$ negative isolates, including 62 carbapenemase producers. Seven different laboratories carried out this method and used the assay for detection of the carbapenemase genes on a selection of 20 isolates.

Results: Both sensitivity and specificity of the multiplex PCR assay was 100\%, as established by results on the strain collection and the inter-laboratory comparisons.

Conclusions: In this study, we present a multiplex real-time PCR that is a robust, reliable and rapid method for the detection of the most prevalent carbapenemases bla OXA-48, $_{1}$ blaVIM, bla $\mathrm{I}_{\mathrm{IMP}}$, bla $\mathrm{a}_{\mathrm{NDM}}$ and $\mathrm{bla}_{\mathrm{KPC}}$ and is suitable for screening of broth cultured rectal swabs and for identification of carbapenemase genes in cultures.
\end{abstract}

Keywords: Real-time multiplex PCR, Carbapenemases, OXA-48, VIM, IMP, NDM, KPC

\section{Background}

Carbapenemase producing Enterobacteriaceae (CPE) are emerging worldwide, and have been implicated in numerous outbreaks [1-3]. Rapid and accurate detection of $\mathrm{CPE}$ is pivotal for adequate antibiotic therapy and infection control, especially in an outbreak setting. The most commonly used phenotypic CPE confirmation tests, the modified Hodge test and the carbapenemase inhibition tests with boronic acid or EDTA/DPA, have several disadvantages, because those tests require an overnight incubation step, do not provide information on the carbapenemase gene, and cannot differentiate OXA-48 producing isolates from $\mathrm{ESBL}$ and/or AmpC producing isolates with decreased permeability [4-7]. Finally, phenotypic detection of CPE

\footnotetext{
* Correspondence: Zeea@maasstadziekenhuis.nl

'Maasstad Laboratory, Molecular Diagnostics Unit, Maasstad Hospital,

Rotterdam, The Netherlands

Full list of author information is available at the end of the article
}

may be difficult because carbapenem MICs may be low (in the susceptible range), especially of OXA-48 producing Enterobacteriaceae. Therefore, genotypic detection of carbapenemase genes is the gold standard, although it only detects a pre-specified set of known genes. Here, we describe a real-time PCR for detection of NDM, KPC, VIM, IMP and OXA-48 genes, which are currently the most prevalent carbapenemases [8]. The main goal of this study was optimization of this real time PCR and to determine the test characteristics on a set of well characterized isolates. An interlaboratory performance comparison of the PCR assay was initiated to investigate its robustness and reliability.

\section{Methods \\ PCR design}

We developed specific real-time PCRs for detection of KPC, VIM, NDM, OXA-48 and IMP. For design of the primers, 
sequence variations of carbapenemase genes published at www.lahey.org/Studies were taken into account, along with synonymous mutations (Additional file 1: Table S1). Detection of CTX-M [9] was included for optional use since it complements OXA-48 for resistance to extended spectrum cephalosporins, a characteristic that is intrinsic to the other carbapenemases. For verification of newly designed PCRs, sequencing was done on larger gene fragments. For NDM sequencing primers 5'-GCGAAAGTCAGGCTGTGTTG3 , and ' 5 '-CATTAGCCGCTGCATTGATG-3', were used, and for IMP sequencing primers 5 '-GGCGGAATA GAGTGGCTTAATTCTC-3', and 5'-CGTACGGTTTAA CAAAACAACCACC-3' For each separate carbapenemase gene, PCR primers and probe concentrations were optimized. Multiplex combinations were compared with single PCRs in presence of the internal control Phocine Herpes Virus (PhHV).

\section{Bacterial strains}

PCRs were optimized and validated using the following control strains: a KPC producing Klebsiella pneumonia, a VIM-2 producing Pseudomonas aeruginosa, an IMP18 producing $P$. aeruginosa, an IMP-28 producing $K$. pneumoniae, a NDM-1 positive $K$. pneumoniae, and an OXA-48 positive $K$. pneumoniae isolate.

Testing of PCR specificity was carried out on the following strain collection. The test collection of 86 isolates, included 58 carbapenemase producing isolates, and 28 carbapenemase negative controls. The 58 carbapenemase positive isolates consisted of $45 \mathrm{~K}$. pneumoniae, 4 E. coli, 3 Enterobacter species, 2 P. mirabilis, 2 Citrobacter species, and $2 \mathrm{P}$. aeruginoasa isolates producing the following carbapenemases: $20 \mathrm{KPC}-2 / 3,4 \mathrm{KPC}$ plus VIM, 21 VIM, 4 NDM-1, 2 IMP, and 7 OXA-48. The 28 carbapenemase negative controls consisted of $10 \mathrm{~K}$. pneumoniae, 2 E. coli and 16 Enterobacter isolates, producing either an ESBL (20 isolates) or an AmpC beta-lactamase (Additional file 2: Table S2). As the reference test for presence of beta-lactamases, PCR and sequencing was used [10].

\section{PCR evaluation}

To determine the sensitivity and specificity of PCR, 86 unrelated test isolates were investigated both from agar plate and broth. Two protocols were followed; protocol 1) simultaneous multiplex detection of OXA-48, VIM, IMP, NDM and KPC. In this PCR reaction, the OXA48 probe was labelled with FAM, and the other carbapenemases with VIC. Protocol 2) 3 multiplex PCRs for identification of respectively OXA-48/CTX-M, VIM/IMP, and NDM/KPC. PhHV was labelled with NED. Fluorescent labels of carbapenemase genes were FAM, and VIC, respectively (Additional file 3: Table S3).

\section{PCR}

Strains were grown on MacConkey agar (Oxoid), and in Brain Heart broth, both supplemented with ertapenem $(0.125 \mathrm{mg} / \mathrm{l})$. One colony was taken from the plate and $50 \mu \mathrm{l}$ from the broth. Both were suspended in $100 \mu \mathrm{l}$ Extraction Solution (SIGMA, E7526). Mixtures were incubated at $95^{\circ} \mathrm{C}$ for 10 minutes, cooled to room temperature, $100 \mu$ Dilution Buffer (SIGMA, D5688) was added and mixed. PCR reactions were carried out using PCRReadyMix $^{\mathrm{TM}}$ (SIGMA, E3004). Amplification was performed on ABI 7500 Real-Time PCR system (LifeTech, Glasgow, UK). The temperature profile included initial denaturation of $4 \mathrm{~min}$. at $94^{\circ} \mathrm{C}$, followed by 50 cycles ( 40 cycles, protocol 2) of $94^{\circ} \mathrm{C}$ for $15 \mathrm{sec}$, and $60^{\circ} \mathrm{C}$ for $1 \mathrm{~min}$.

\section{Results}

\section{Evaluation of PCR}

PCRs on serial dilutions of template showed Cq (Cycle threshold) values ranging from 19.5-41.5 for single PCRs, and $\mathrm{Cq}$ values of 20.2-46.3 for multiplex reactions. The mean difference in $\mathrm{Cq}$ value between single and multiplex reactions was 0.9 , with a tendency towards an increased difference with decreasing template concentrations. Mixing of templates did not affect $\mathrm{Cq}$ values. The lower limit of detection was approximately 10 colony forming units. The amplification efficiencies in multiplex format ranged from $85 \%$ for VIM to $82 \%$ for IMP (Figure 1). We concluded that the PCRs were compatible.

The PCR assay according to protocol 1 was carried out on the test collection of 86 strains (Additional file 2: Table S2). All carbapenemase producing isolates were positive in the PCR with Cq values ranging from 15.4 to 23 for isolates from agar, and Ct values 20.1 to 28 from broth cultures (Figure 2A), corresponding to a sensitivity of $100 \%$. All 26 carbapenemase negative control isolates were negative in the PCR, corresponding to a specificity of $100 \%$.

To evaluate the capacity of the real-time PCR to identify carbapenemase genes, protocol 2 was used. Twentyfive strains were PCR positive for VIM, 2 for IMP, 4 for NDM, 24 for KPC, 7 for OXA-48 and 18 for CTX-M. Four strains were positive for both VIM and KPC and 2 strains both for OXA-48 and CTX-M (Figure 2B). Ct values ranged from 15.1-22.5 (VIM), 13.2-20.6 (KPC), 17.8-20.1 (NDM), 16.8-23.8 (OXA-48) and, 14.7-17.2 (CTX-M). The results were $100 \%$ concordant with listed strain characteristics.

\section{Multi-centre comparison}

The PCR assay (protocol 2) was tested on a selection of 20 strains (Table 1) by 7 different laboratories. Different PCR platforms were used; ABI7500 (Life Technologies), RotorgeneQ (Qiagen), Biorad CFX96 (Bio-Rad), and Lightcycler 480 (Roche). Also different DNA polymerases 


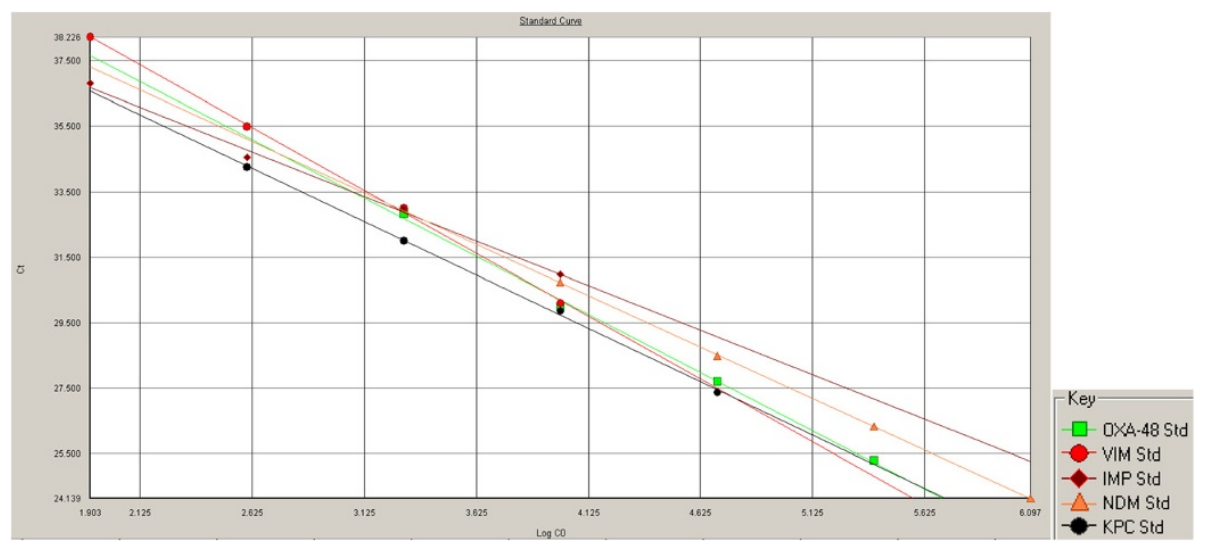

Figure 1 Standard curve of multiplex PCR. Inferred efficiencies of multiplex amplification range from 82\% for IMP, to $85 \%$ for VIM.

were used; PCR ReadyMix (Sigma), Platinum ${ }^{\circ}$ Multiplex PCR Master Mix (Life Technologies), Fast start master (Roche), and Master Mix for PCR (Bio-Rad). All laboratories correctly detected the targeted carbapenemase genes, and no false positive results were found. With exception of CTX-M, which was not tested, or partially tested as is shown in Table 1, results of the multi-centre comparison were all concordant and again, the results were 100\% concordant with listed strain characteristics.

Thus, both inter- and intra-laboratory, PCR sensitivity and specificity corresponds to $100 \%$. Two laboratories also subjected the 20 control strains to Check-points PCR assay (laboratory 3) and Check-MDR Carba Assay (laboratory 6). The results were concordant with our PCR assay, except that In the Check-MDR Carba Assay IMP-28 was missed.

\section{Discussion}

This assay has several advantages. First, it is able to detect the five most prevalent carbapenemases, whereas previously published real-time PCRs for detection of carbapenemases were designed to detect either exclusively KPC, or exclusively NDM, or a combination of GES, IMI/NMC, KPC, OXA-48 and SME [11-14]. Second, the good performance of the assay when using pre-cultured broth, makes this method suitable for detection of carbapenemases in clinical swabs.

The PCR assays described here were designed to predominantly detect OXA-48 in the follow-up of an outbreak. The PCR assay according to protocol 1 therefore can only detect if one of the other carbapenemases (except OXA-48) is present or not. The probes of the other carbapenemases can also be differentially

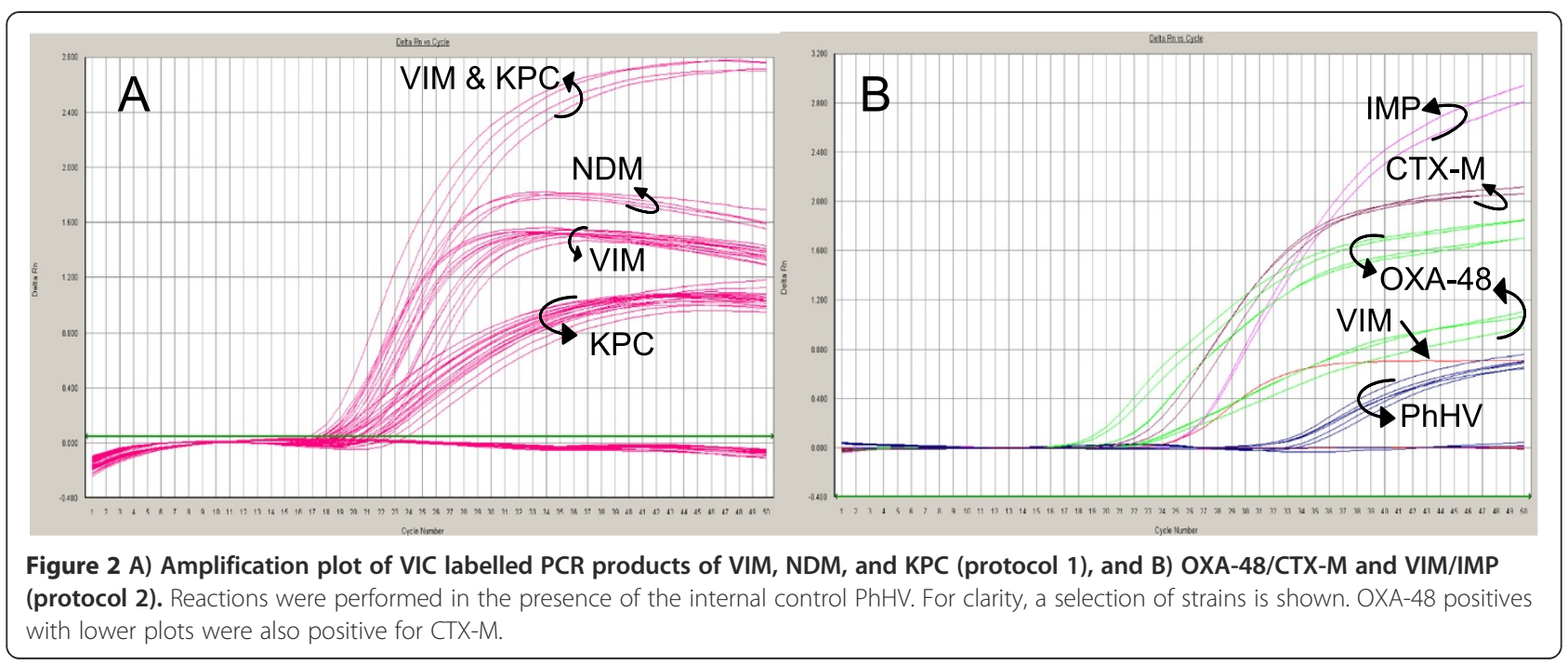


Table 1 Multi-centre comparison of the PCR assay for detection of carbapenemases, and the PCR platforms, and DNA polymerases used

\begin{tabular}{|c|c|c|c|c|c|c|c|}
\hline Strain ID & Laboratory1 & Laboratory2 & Laboratory3 $^{1}$ & Laboratory4 ${ }^{1}$ & Laboratory $5^{2}$ & Laboratory $6^{2}$ & Laboratory7 $^{2}$ \\
\hline OXA 8-90 & OXA-48 & OXA-48 & OXA-48 & OXA-48 & OXA-48 & OXA-48 & OXA-48 \\
\hline OXA 8-17 & OXA-48/CTX-M & OXA-48/CTX-M & OXA-48/CTX-M & OXA-48/CTX-M & OXA-48 & OXA-48 & OXA-48 \\
\hline S 4-2 & OXA-48/CTX-M & OXA-48/CTX-M & OXA-48/CTX-M & OXA-48/CTX-M & OXA-48 & OXA-48 & OXA-48 \\
\hline S3-60 & OXA-48/CTX-M & OXA-48/CTX-M & OXA-48/CTX-M & OXA-48/CTX-M & OXA-48 & OXA-48 & OXA-48 \\
\hline GR-21/PM-302 & VIM & VIM & VIM & VIM & VIM & VIM & VIM \\
\hline GR-04/KP-69 & VIM/KPC & VIM/KPC & $\mathrm{VIM} / \mathrm{KPC}$ & VIM/KPC & VIM/KPC & VIM/KPC & VIM/KPC \\
\hline GR-38/KP-139 & VIM & VIM & VIM & VIM & VIM & VIM & VIM \\
\hline GR-31/KP-956 & VIM/KPC & VIM/KPC & VIM/KPC & VIM/KPC & VIM/KPC & VIM/KPC & VIM/KPC \\
\hline GR-23/KP-385 & KPC & KPC & KPC & KPC & KPC & KPC & KPC/imp* \\
\hline New York-11 & KPC & KPC & KPC & KPC & KPC & KPC & KPC \\
\hline New York-3 & KPC & $\mathrm{KPC}$ & KPC & KPC & KPC & KPC & $\mathrm{KPC}$ \\
\hline JS022 & NDM/CTX-M & NDM/CTX-M & NDM/CTX-M & NDM/CTX-M & NDM & NDM & NDM \\
\hline RC-89 & NDM/CTX-M & NDM/CTX-M & NDM/CTX-M & NDM/CTX-M & NDM & NDM & NDM \\
\hline S 3-62 & IMP & IMP & IMP & IMP & IMP & IMP & IMP \\
\hline S5-36 & IMP & IMP & IMP & IMP & IMP & IMP & IMP \\
\hline RC-79 & CTX-M & CTX-M & CTX-M & CTX-M & negative & negative & negative \\
\hline EIE-UMC-1 & CTX-M & CTX-M & negative & negative & negative & negative & negative \\
\hline $\mathrm{RC}-8$ & negative & negative & negative & negative & negative & negative & negative \\
\hline$R C-16$ & negative & negative & negative & negative & negative & negative & negative \\
\hline GR-07/KP-3878 & negative & negative & negative & negative & negative & negative & negative \\
\hline \multirow[t]{2}{*}{ thermocycler } & Biorad CFX & LC 480 & $A B I 7500$ & $A B \mid 7500$ & LC 480 & $A B \mid 7500$ & LC 480 \\
\hline & & & Biorad CFX & & & & \\
\hline polymerase & Sigma & Roche & $\mathrm{ABI}$ & $A B \mid$ & Roche & N-Amp & Roche \\
\hline
\end{tabular}

${ }^{1}$ Only CTX-M group I PCR was performed.

${ }^{2}$ No CTX-M PCR was performed.

*: Background IMP was detected, and scored as inconclusive by laboratory 7.

labelled, making it possible to simultaneously detect and identify the genes. This is however limited to 3-4 different labels in addition to that of the internal control, and depends on the spectral specifics of the type of thermocycler.

Multi-centre evaluation of the assay showed concordant results, which demonstrates that the test is robust and can be performed in different laboratories using different amplification platforms and/or DNA polymerases.

The PCR detection of the IMP gene still requires attention. Check-Points also has withdrawn detection of IMP from their latest kit. Although we designed two probes that in theory can detect all IMP variants, we have not been able to test whether this is indeed the case. This will be subject for future study.

An intrinsic limitation of this type of assay is that new carbapenemase families or new variants of known families may not be detected. The flexibility of the system presented here, however, allows easy adaptation. For example, detection of other genes e.g. PER or GES, might be added to this multiplex PCR.

\section{Conclusions}

The multiplex real-time PCR described here is a robust, reliable and rapid method for detection of the most prevalent carbapenemase genes bla $\mathrm{OXX}_{-48}, \mathrm{bla}_{\mathrm{VIM}}$, bla $_{\mathrm{IMP}}$, $\mathrm{bla}_{\mathrm{NDM}}$ and $\mathrm{bla}_{\mathrm{KPC}}$.

\section{Additional files}

Additional file 1: Table S1. DNA sequences of primers and probes directed against five carbapenemase genes, CTX-M group I-V, and the internal control Phocine Herpes Virus (PhHV) [15]

Additional file 2: Table S2. Investigated isolates and their characteristics. Additional file 3: Table S3. PCR protocol; components and

concentration of primers and probes for separate PCRs for screening and determination.

Competing interests

The authors declare that they have no competing interests.

\section{Authors' contributions}

AZ conceived of the study, participated in the design, and drafted the manuscript. LR carried out the molecular genetic studies. GB and JMO participated in the design and coordination. The remaining authors 
coordinated testing and helped to draft the manuscript. All authors read and approved the final manuscript.

\section{Acknowledgements}

The OXA-48 producing K. pneumoniae was kindly provided by Prof. $\mathrm{dr}$. Patrice Nordmann, University of Fribourg, Switzerland.

\section{Author details}

${ }^{1}$ Maasstad Laboratory, Molecular Diagnostics Unit, Maasstad Hospital, Rotterdam, The Netherlands. ${ }^{2}$ Department of Medical Microbiology, University Medical Center Utrecht, Utrecht, The Netherlands. ${ }^{3}$ Molecular Diagnostics, Jeroen Bosch Hospital, 's-Hertogenbosch, The Netherlands. ${ }^{4}$ Molecular Biology Laboratory, Slotervaart Hospital, Amsterdam, The Netherlands. ${ }^{5}$ Laboratory for Microbiology and Public Health, Enschede, The Netherlands. ${ }^{6}$ Department of Medical Microbiology and Infectious Diseases, Erasmus MC, Rotterdam, The Netherlands. "Isala clinics, Laboratory for Medical Microbiology and Infectious Diseases, Zwolle, The Netherlands.

${ }^{8}$ Medical Centre Alkmaar, Alkmaar, The Netherlands.

Received: 3 September 2013 Accepted: 24 December 2013

Published: 14 January 2014

\section{References}

1. Cuzon G, Ouanich J, Gondret R, Naas T, Nordmann P: Outbreak of OXA-48-positive carbapenem-resistant Klebsiella pneumoniae isolates in France. Antimicrob Agents Chemother 2011, 55:2420-2423.

2. Goren MG, Chmelnitsky I, Carmeli Y, Navon-Venezia S: Plasmid-encoded OXA-48 carbapenemase in Escherichia coli from Israel. J Antimicrob Chemother 2011, 66(3):672-673.

3. Kitchel B, Rasheed JK, Patel JB, Srinivasan A, Navon-Venezia S, Carmeli Y, Brolund A, Giske CG: Molecular Epidemiology of KPC-Producing Klebsiella pneumoniae Isolates in the United States: Clonal Expansion of Multilocus Sequence Type 258. Antimicrob Agents Chemother 2009, 53(8):3365-3370.

4. Girlich D, Poirel L, Nordmann P: Value of the modified hodge test for detection of emerging carbapenemases in Enterobacteriaceae. J Clin Microbiol 2012, 50:477-479.

5. Giske CG, Gezelius L, Samuelsen Ø, Warner M, Sundsfjord A, Woodford N: A sensitive and specific phenotypic assay for detection of metallo- $\beta$-lactamases and KPC in Klebsiella pneumoniae with the use of meropenem disks supplemented with aminophenylboronic acid, dipicolinic acid and cloxacillin. Clin Microbiol Infect 2011, 17(4):552-556.

6. Pasteran F, Mendez T, Guerriero L, Rapoport M, Corso A: Sensitive screening tests for suspected class A carbapenemase production in species of Enterobacteriaceae. J Clin Microbiol 2009, 47:1631-1639.

7. Tsakris A, Poulou A, Pournaras S, Voulgari E, Vrioni G, Themeli-Digalaki K, Petropoulou D, Sofianou D: A simple phenotypic method for the differentiation of metallo-beta-lactamases and class A KPC carbapenemases in Enterobacteriaceae clinical isolates. J Antimicrob Chemother 2010, 65(8):664-671.

8. Cohen Stuart J, Leverstein-Van Hall MA, on behalf of members of the Dutch Working Party on the Detection of Highly Resistant Microorganisms: Guideline for phenotypic screening and confirmation of carbapenemases in Enterobacteriaceae. Int J Antimicrob Agents 2010, 36:205-210.

9. Van Hannen EJ: Nieuwegein, The Netherlands: St. Antonius Hospital. e. vanhannen@antoniusziekenhuis.nl. Personal communication.

10. Voets GM, Fluit AC, Scharringa J, Cohen Stuart J, Leverstein-van Hall MA: A set of multiplex PCRs for genotypic detection of extended-spectrum beta-lactamases, carbapenemases, plasmid-mediated AmpC 1-lactamases and OXA-lactamases. Int J Antimicrobial Agents 2011, 37:356-359.

11. Chen L, Mediavilla JR, Endimiani A, Rosenthal ME, Zhao Y, Bonomo RA, Kreiswirth BN: Multiplex Real-Time PCR Assay for Detection and Classification of Klebsiella pneumoniae Carbapenemase Gene (blakpd) Variants. J Clin Microbiol 2011, 49(2):579-585.

12. Cole JM, Schuetz AN, Hill CE, Nolte FS: Development and Evaluation of a Real-Time PCR Assay for Detection of Klebsiella pneumoniae Carbapenemase Genes. J Clin Microbiol 2009, 47(2):322-326.
13. Naas T, Ergani A, Carrër A, Nordmann P: Real-Time PCR for Detection of NDM-1 Carbapenemase Genes from Spiked Stool Samples. Antimicrob Agents Chemother 2011, 55(9):4038-4043.

14. Poirel L, Walsh TR, Cuvillier V, Nordmann P: Multiplex PCR for detection of acquired carbapenemase genes. Diagn Microbiol Infect Dis 2011, 70:119-123.

15. Van der Bij AK, Mol M, Van Westreenen M, Goessens WHF, Pitout JDD: The laboratory diagnosis of Pseudomonas aeruginosa that produce metallo- beta -lactamases in a Dutch tertiary care centre. Scand $J$ Infect Dis 2011, 43(8):596-602.

doi:10.1186/1471-2334-14-27

Cite this article as: van der Zee et al: Multi-centre evaluation of real-time multiplex PCR for detection of carbapenemase genes OXA-48, VIM, IMP, NDM and KPC. BMC Infectious Diseases 2014 14:27.

\section{Submit your next manuscript to BioMed Central and take full advantage of:}

- Convenient online submission

- Thorough peer review

- No space constraints or color figure charges

- Immediate publication on acceptance

- Inclusion in PubMed, CAS, Scopus and Google Scholar

- Research which is freely available for redistribution

Submit your manuscript at www.biomedcentral.com/submit
C Biomed Central 